\title{
EVIDENCE FOR PHYSICAL AGING OF PERIODIC COMETS
}

\author{
Ľ. KRESÁK \\ Astronomical Institute, Slovak Academy of Sciences \\ 84228 Bratislava, Czechoslovakia
}

\begin{abstract}
Observational evidence for the aging processes in periodic comets is reviewed. This includes progressive changes of the comets' absolute brightness, sudden destructive events (outbursts and splitting of cometary nuclei), temporary intermissions in activity, total disappearance, and existence of asteroidal objects moving in cometary orbits. Indirect statistical evidence is provided by the equilibrium between the aging rate and the net injection of comets into the inner planetary system. All of this information is consistent with typical active lifetimes of 200 to 500 revolutions at small perihelion distances $(\mathrm{q}<3 \mathrm{AU})$. The active lifetimes are sometimes intermitted by dormant periods, which tend to occur especially during the latest phases of evolution. Splits and outbursts do not seem to have a decisive statistical effect on the survival time. Some periodic comets evolve into inactive asteroid-like objects, but the question of whether these still contain some supply of volatiles, and thus can renew their activity, remains open.
\end{abstract}

\section{Introduction}

The aging processes in comets consist of: (1) progressive loss of gas and dust at each passage near the Sun, (2) occasional outbursts and splitting of their nuclei, and (3) changes in their size, structure, and thermal properties - in particular, in their surface crusting and porosity.

Some components of the mass loss, especially the outflow of gas and finer dust, can be measured with confidence. However, the contribution of larger solid particles is still rather uncertain (McDonnell et al. 1986, Crifo 1987, Green et al. 1987). If the orbit is of short period and passes close to the orbit of the Earth, the associated meteor stream provides some information on this point, but a great deal of extrapolation is necessary (Hajduk 1986). For the aging rate, the total relative mass loss is essential, and its determination requires knowledge of the comet's mass. So far, only indirect order-ofmagnitude estimates of this quantity are available. They are mainly based on the operation of nongravitational effects that can be detected in the motion of short-period comets observed at a number of apparitions (Marsden 1985), and require model assumptions of some numerical parameters (Rickman 1986, Rickman et al. 1987). Observations of comet splitting provide information on their occurrence rate (Whipple 1978a, Kresák 1981a), but the relative masses of the separated components, which are essential for determination of 
their survival rate, are difficult to ascertain (Sekanina 1982). The degree of surface crusting has been directly observed only on P/Halley (Whipple 1986 and 1987, Keller 1987). Asteroid-like objects moving in typical cometary orbits (Rickman 1985, Milani et al. 1989, Wetherill, this book) indicate that there very probably are also cases of complete crusting, or complete depletion of volatiles, as the final evolutionary stage.

A long-period comet is observable only during one passage near the Sun, for several weeks to a few years. Within this time span, the comet exhibits major changes associated with the varying heliocentric distance, but, as a rule, no progressive changes. There are only a few observations of untimely disappearance (Kresák 1984, Sekanina 1984), which suggest a mean active lifetime of about 20 revolutions for a perihelion distance of $1 \mathrm{AU}$ (Kresák 1985). It is just the early disappearance that makes the orbits and dynamical ages of these comets indeterminate. An important characteristic of the statistical data is a high proportion of new comets, passing between the planets for the first time (see Marsden and Roemer 1982, Fernández 1985a, Kresák 1987a). This indicates that most of these comets do not survive more than one such passage as active objects.

An indirect approach to the problem consists of statistical modelling of the dynamical evolution of the whole comet system (Everhart 1976 and 1977, Rickman and Vaghi 1976), and comparison of the outcome with the observed state (Rickman and Vaghi 1976, Fernández 1981, Weissman 1982 and 1986). While the number of objects whose orbits are determined with sufficient accuracy is large enough-about 300 long-period and 150 short-period comets - the main problem is the choice of the starting conditions. The origin of all the objects near the outer boundary of the Oort Cloud is usually assumed. However, it appears that some comets-in particular, the periodic comets-may have originated in the flattened inner Oort cloud, or Kuiper Belt (Kuiper 1951, Whipple 1964 and 1972, Joss 1973, Bailey 1983, Fernández and Ip 1983, Clube and Napier 1984, Bailey et al. 1986, Duncan et al. 1987), and this would make equilibrium on longer time scales questionable (Hills 1981, Bailey et al. 1986, Weissman 1986).

Short-period comets are the only objects on which the aging can be directly observed, since they can be followed over a number of revolutions. A list of such objects with revolution periods of less than 20 years (as of January 1,1989 ) is presented in Table 1. $T_{F}$ denotes the year of the first apparition (i.e., the year of the first observed perihelion passage) and $T_{L}$ that of the last one. $N_{A}$ is the number of apparitions and $N_{P}$ the number of perihelion passages within this time span; the difference $\mathrm{N}_{P}-\mathrm{N}_{A}$ indicates the number of missed returns from which no observations are available. The total of 785 perihelion passages is on the same order of magnitude as the current estimates of typical lifetimes of short-period comets, but only 10 individual objects contribute to this total by more than $2 \%$, and only one of them, P/Encke, by more than $5 \%$.

Changes of the observational data base with time are illustrated in Figure 1. In addition to the increase of the number and quality of observations, the number of known periodic comets has more than doubled during the last 50 years, that of comets of more than one apparition during the last 40 years, and that of comets of more than four apparitions during the last 30 years.

Unfortunately, results obtained by modern sophisticated observing techniques have very limited application when direct comparison with observations made long ago is required. There are also two reasons why conclusions concerning periodic comets cannot be generalized to all comets. The first is the already mentioned possibility of their different place of origin, which could have made their compositional and structural properties 
Table 1. Comets of $P<20$ Years in Order of the Number of Perihelion Passages Since the First Observation

\begin{tabular}{|lcccc||}
\hline Comet & $\mathrm{T}_{\mathrm{F}}$ & $\mathrm{T}_{\mathrm{L}}$ & $\mathrm{N}_{\mathrm{A}}$ & $\mathrm{N}_{\mathrm{P}}$ \\
\hline P/Encke & 1786 & 1987 & 54 & 62 \\
P/Grigg-Skjellerup & 1808 & 1987 & 16 & 38 \\
P/Pons-Winnecke & 1819 & 1983 & 19 & 29 \\
P/Tuttle-Giacobini-Kresák & 1858 & 1978 & 6 & 23 \\
P/Tempel 2 & 1873 & 1988 & 18 & 23 \\
P/DeVico-Swift & 1844 & 1965 & 3 & 21 \\
P/D'Arrest & 1851 & 1982 & 14 & 21 \\
P/Tempel 1 & 1867 & 1983 & 7 & 20 \\
P/Faye & 1843 & 1984 & 18 & 20 \\
P/Peters-Hartley & 1846 & 1982 & 2 & 18 \\
\hline 10 comets, $\mathrm{NP}_{\mathrm{P}}>17$ & 1786 & 1988 & 157 & 275 \\
19 comets, $\mathrm{NP}_{\mathrm{P}}=10$ to 16 & 1772 & 1988 & 148 & 235 \\
23 comets, $\mathrm{N}_{\mathrm{P}}=5$ to 9 & 1846 & 1988 & 122 & 151 \\
29 comets, $\mathrm{NP}=2$ to 4 & 1916 & 1988 & 74 & 76 \\
48 comets, $\mathrm{NP}=1$ & 1770 & 1988 & 48 & 48 \\
\hline \hline Total, 128 comets & & & & 785 \\
\hline \hline
\end{tabular}




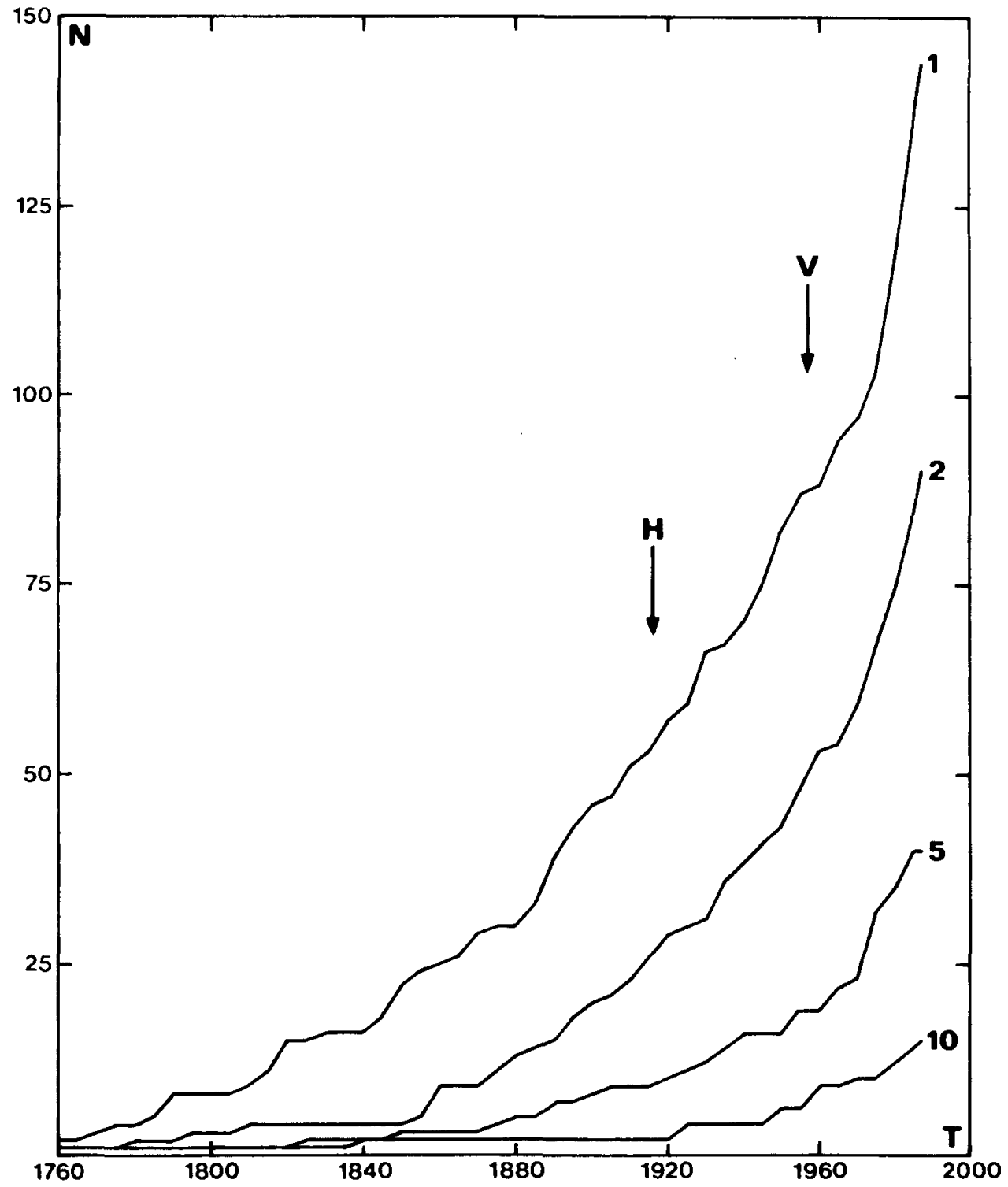

Figure 1. Increase of the number of periodic comets observed at their first, second, fifth, and tenth apparitions, as a function of time. The end points of the data sets available to Holetschek $(\mathrm{H})$ and Vsekhsvyatskij (V) are marked by arrows (Kresák and Kresáková 1989a). 
different from the very beginning. The second is the fact that these comets have undergone a long and complicated dynamical evolution, with the perihelion distance and insolation regime changed many times by planetary perturbations. This process must have introduced a selection of more resistive objects, and possibly also some changes in their structure. Thus, the sample of short-period comets is far from typical for the whole comet population, which is a billion times more numerous.

The past dynamical evolution of the Halley-type comets (with periods of 20 to 200 years, all inclinations, and Tisserand invariants with respect to Jupiter of less than 2) was substantially different (see Carusi et al. 1985a and 1987). Therefore, in the following sections, we shall only deal with the short-period comets with periods less than 20 years, sometimes referred to as the "Jupiter family," as summarized in Table 1.

\section{Progressive Brightness Variations}

Determination of the progressive brightness variations of periodic comets, as a manifestation of their aging, requires a very extensive data base covering hundreds of individual apparitions. A pioneering work in collecting such data was done by Holetschek (1916 and 1917), Bobrovnikoff (1941 and 1942), and especially Vsekhsvyatskij (1958). In a well-known monograph followed by five later supplements (Vsekhsvyatskij 1966, 1967, and 1979, Vsekhsvyatskij and Ilchishina 1974, Andrienko and Karpenko 1987), Vsekhsvyatskij and his colleagues have published not only descriptions of all comet apparitions, but also lists of their computed absolute magnitudes. These implied a rapid secular fading as a common property of all periodic comets. Table 2 shows that the mean fading rates, as determined by different investigators for different comet samples and different time spans, were rather consistent: about 0.3 magnitude per revolution, or 4 to 5 magnitudes per century.

However, important contradictions soon appeared. Forward extrapolation of the brightness trends toward complete extinction yielded unacceptably early death dates (Whipple and Douglas-Hamilton 1966), which were ruled out by continuing apparitions of the respective comets. Backward extrapolation for the bright and best-documented $\mathrm{P} /$ Encke would definitely require records of spectacular apparitions in the medieval and ancient chronicles, but none could be identified (Whipple and Hamid 1972). Arguments pointing to major systematic time-dependent errors in the magnitude estimates, and their explanation by observational and instrumental effects, were presented by Kresák (1966 and 1974) and confirmed by Meisel and Morris (1976 and 1982). It was also shown that acceptance of the rapid fading would require an early disappearance not only of the individual objects, but also of the system of short-period comets as a whole (Kresák 1974 and 1982). It must be emphasized that some of the authors quoted in Table 2 -in particular, Hughes and Daniels (1983) and Dobrovolsky et al. (1986)-used their computed fading rates just to demonstrate a discrepancy of nearly two orders of magnitude between the observed variations and those that they theoretically predicted, and preferred.

The whole problem rests in the incompatibility of observations from different epochs, influenced by the development of instrumentation and observing techniques. With the changing brightness threshold accessible (Kresák 1982, Figure 4), observations at a lower absolute brightness and geometrically unfavorable perihelion passages became possible. And it is exactly these cases that require the greatest instrumental corrections, 
Table 2. Comets of $\mathrm{P}<20$ Years: Mean Secular Variations of Absolute Magnitudes

Source

$\Delta \mathrm{H}$ Per Revolution

Sekanina (1964)

$0.33 \pm 0.04$

Vsekhsvyatskij (1972)

$0.35 \pm 0.05$

Svoreñ (1979)

$0.22 \pm 0.06$

Hughes and Daniels (1983)

$0.30 \pm 0.06$

Dobrovolsky et al. (1986)

$0.38 \pm 0.05$

Guliev and Bajramov (1988)

$0.26 \pm 0.04$

Kresák and Kresáková (1989)

$0.02 \pm 0.01$

always negative. The corrections reflect the fact that a large telescope records only the inner condensation of the coma, and its outer parts are sometimes even subtracted as a part of the background.

Some investigators have used instrumental corrections as a function of the telescope aperture (Bobrovnikoff 1941, Morris 1973). A weak point of this procedure is that the apparent brightness also varies with changes of the magnification in the same visual telescope, or the exposure time in the same photographic telescope. Therefore, another approach was used by Kresák and Kresáková (1987a), with the correction expressed as a function of the recorded magnitude. This function was determined in two different ways, with very good mutual agreement. In order to minimize the corrections, the absolute brightness of each comet apparition was determined from the peak of the absolute lightcurve.

Application of these corrections reduces the mean fading rate by more than one order of magnitude, to $0.20-0.025$ magnitude per revolution. For comets of smaller perihelion distance, $\mathrm{q}<1.5 \mathrm{AU}$, the systematic trend of about 0.010 magnitude per revolution is hardly discernible, being overlapped by much larger irregular fluctuations. For comets of $\mathrm{q}>1.5 \mathrm{AU}$, the mean fading rate is statistically significant, about $0.04-0.05$ magnitude per revolution. However, a part of this variation is due to the fact that some of these comets were discovered immediately after a significant reduction of their perihelion distance by Jupiter. Such reduction tends to be accompanied by a relative brightening for a few apparitions. The apparent paradox that comets that receive more solar irradiation tend to fade less rapidly is probably due to a combination of the surface crusting and purging processes (Shulman 1972, Whipple 1978b, Fernández 1985b). Since an 
appreciable reduction of the perihelion distance requires, on the average, a longer and more complicated orbital evolution, a selection of larger and more resistive objectives may also be involved. More details on the brightness changes can be found in the paper by Kresák and Kresáková (1989a).

A good example of the operation of the instrumental effects is P/Grigg-Skjellerup, a comet with the second highest number of returns since its first apparition, after P/Encke. Its first apparition of 1808 was identified only recently (Kresák 1987b), and its description by Pons (1829) indicated an apparent magnitude between 7.0 and 8.0. The lower limit corresponds exactly to the mean absolute magnitude in this century. On the other hand, the secular variation based on Vsekhsvyatskij's uncorrected data would require an entirely unacceptable apparent magnitude of $-0.5 \pm 1.0$.

\section{Equilibrium Between Source and Sink}

Another approach to the problem of comet aging is of a statistical nature. It is based on the comparison of the physical and dynamical evolution of short-period comets, assuming an equilibrium between those captured into small-q orbits and the sum of those ejected or faded away. On longer time scales, this assumption would be questionable, in view of the possible comet showers (Hills 1981, Bailey et al. 1986), and of the insufficiency of the current dust production by periodic comets to maintain the zodiacal cloud (Whipple 1967, Delsemme 1976, Kresák and Kresáková 1987b). However, on the time scale of the few centuries that are covered by comet observations and reliable orbit integrations, the assumption of a statistical equilibrium appears sound.

This approach was applied by Fernández (1985b) to the orbit integrations of 109 periodic comets over 800 years by Carusi et al. (1985a). Fernández's main conclusion was that the prevalence of captures over ejections reflected by these data can be explained by assuming a mean lifetime of about 850 revolutions. A more detailed comparison, taking into account the absolute magnitudes of the individual comets and their osculating orbits for the epochs 1900 and 2000, was made by Kresák and Kresáková (1989b). The results agree very well with those mentioned in Section 2-not only as to the mean fading rate of $0.020-0.025$ magnitude per revolution, but also as to the difference between comets with perihelion distances smaller and larger than 1.5 AU. Under the simplifying assumption that the dimensions of the active areas on the nuclear surface decrease linearly with time (which is equivalent to an active layer of constant depth being removed at each perihelion passage), and that the present observations are distributed at random within the active lifetimes of individual comets, a fading rate of 0.022 magnitude per revolution would correspond to an active lifetime of 200 revolutions. This is about one-half of the estimate based on the statistics of comet disappearances (Kresák 1981b), and one-fourth of the value found by Fernández (1985b). A longer total lifetime-including dormant periodswould result in the presence of temporary intermissions in activity, to be discussed in Section 5. 


\section{Splitting and Outbursts}

The progressive aging of some comets may be accelerated by sudden destructive events: splitting of their nuclei, or strong enhancement of the mass loss during their outbursts.

Observed cases of splitting of short-period comets occur, on the average, about once per 30 years. This implies one split per 80 revolutions, as compared with one per 12 revolutions for the long-period comets (Kresák 1981a). However, even this lower occurrence rate requires that a majority of short-period comets would split at least once during their active lifetime.

The impact of the splits on the aging process depends primarily on the relative mass of the separated components, and can be checked by observations at later returns. Both of these sources of information point to a broad variety in the resulting reduction of the survival time. The mass ratios, based on the measured relative deceleration of the split components, have been estimated by Sekanina (1982) using different models. The ratio was found to be smallest, on the order of $1: 10$, for P/Biela; this was indeed the only case in which both components were observed at two successive perihelion passages, and then disappeared completely. The next example is $\mathrm{P} / \mathrm{Brooks} 2$, which experienced a tidal breakup at a close encounter with Jupiter. A multiple nucleus was observed during its discovery apparition in 1889 , and the smaller components seem to have comprised less than 5\% of the total mass (Sekanina 1982). The smaller components never reappeared again, but the primary was observed at 13 apparitions since then, without any unusual fading (Kresák and Kresáková 1989a). The primary of P/Taylor, with the separated mass less than $1 \%$ in 1916, was, after 8 missed returns, rediscovered in 1977 and again in 1984, 1.5 magnitude fainter than at the time of splitting. P/DuToit-Hartley, the duplicity of which was recorded only in 1982, became 2 magnitudes brighter in 1987. All of these data refer to the absolute brightness. P/Giacobini was never found after its discovery apparition, when it had a double nucleus, although it should have reached magnitude 8 to 9 in 1916 and 1929. P/Van Biesbroeck and P/Neujmin 3, which were produced by splitting of a common parent body 140 years ago (Carusi et al. 1985b), have survived 11 and 12 revolutions, respectively, since then. Their absolute magnitudes indicate a probable mass ratio of about 10:1. The smaller object, P/Neujmin 3, was not discovered in 1982. However, since it should not have become brighter than magnitude 20 at that time, there is no reason for supposing its disappearance.

Thus, the record of the splits of comets is rather poor and controversial. Anyway, their statistical effect on the survival times of periodic comets does not seem to be decisive.

The phenomenon of cometary outbursts (Hughes 1975 and this book) is wellknown thanks to the peculiar P/Schwassmann-Wachmann 1, in which the outbursts repeat many times per revolution at considerable heliocentric distance (Whipple 1980). Other conspicuous examples are $\mathrm{P} / \mathrm{Holmes}$ and $\mathrm{P} / \mathrm{Tuttle-Giacobini-Kresák,} \mathrm{for} \mathrm{which} \mathrm{the}$ outburst amplitudes exceeded 7 magnitudes. This corresponds to a brightness change by three orders of magnitude and to a mass loss equivalent to that during more than 10 whole revolutions. A peculiar property of these extreme outbursts is that they tend to recur in pairs spaced by one to two months. This repetition, statistically very significant, has led Whipple (1983a, 1984) to propose a triggering mechanism of an impact by a satellite of the comet. P/Schwassmann-Wachmann 1 also displays a tendency for a similar recurrence. However, here the situation is different in that the outbursts appear very often with a wide 
range of amplitudes. This contradicts the explanation that we are dealing with longer-lived satellites.

The outbursts of $\mathrm{P} / \mathrm{Holmes}$ occurred during its discovery apparition in 1892 , and the comet has already made 13 perihelion passages since then, with a very moderate progressive brightness decrease. As the comet remained unobserved for perihelion passages between 1906 and 1964, irregular brightness variations cannot be ruled out. The outbursts of P/Tuttle-Giacobini-Kresák in 1973 occurred during the 22nd perihelion passage after its first discovery, and its absolute brightness at all other apparitions was rather stable. They were followed by peculiar nongravitational effects, resembling those that have preceded the disappearance of other comets (Marsden 1985). The failure of rediscovery in 1984 does not yield persuasive evidence of P/Tuttle-Giacobini-Kresák's extinction, because it was unfavorably situated and should not have become brighter than magnitude 16.

Thus, the role of the outbursts in the aging of comets appears to be statistically less significant than that of splitting. The frequency of outbursts with amplitudes exceeding 5 magnitudes is about the same as the frequency of splitting into clearly discernible components. In this respect, the behavior of long-period comets is different. There are no records of outbursts with such large amplitudes, but about 20 cases of observed splitting. The repetition of outbursts in the same periodic comets is indicative of some specific property of these objects; this property, however, is not yet understood.

\section{Extinction and Reactivation}

The simplest evidence of the limited lifetimes of comets is the disappearance of some of them. The most famous example was P/Biela, which was observed at four apparitions from 1772 to 1832 as a normal comet, was observed in 1846 and 1852 as a double comet, and has never been found again during its 20 predicted returns since then. The disappearance of the comet was followed by the Bielid/Andromedid meteor showers in 1872 and 1885, with the highest concentration of meteor particles ever observed from the Earth (Kresák 1980). For a long time, P/Biela has supported the hypothesis that the comet lifetimes are very limited and end with a sudden complete disintegration into meteor particles.

From the statistical point of view, this case was exceptional and somewhat misleading. Among the more than 100 short-period comets discovered since then, no similar succession of events has been observed, although many of the comets have been lost. The failure of a comet recovery, however, need not be associated with the comet's actual disappearance. Many discoveries are made under unusually favorable observing conditions-in particular, when the comet passes its perihelion near opposition with the Sun. If the comet is not observed long enough, the prediction of subsequent returns becomes unreliable, and we have to wait until the next very favorable return for an independent rediscovery. In the meantime, the orbit can change by planetary perturbations, and a rediscovery can become hopeless.

A statistical summary of the history of comet losses and rediscoveries is presented in Table 3 for all comets of $P<20$ years and in Table 4 for those of more than one apparition. The comets are divided into eight equally numerous groups according to the dates of their first observation. The state at the end of each period is also given. The 
losses are dated from the second perihelion passage at which the comet remained unobserved. Their cumulative numbers include those comets that have not been recovered during the two last returns before the end of the respective period. At present, these are $\mathrm{P} /$ Biela, P/Brorsen, P/Tempel-Swift, P/Neujmin 2, P/Gale, P/DeVico-Swift, P/PerrineMrkos, and twelve additional one-apparition comets.

Both tables demonstrate the rapidly decreasing proportion of lost comets with the progress in observing and computing techniques. The highest number of losses dates back to the beginning of this century, when early photographic techniques were still unable to cope with the number of comet discoveries at the end of the 19th century, for which only positional measurements of low accuracy from short time spans were available. Around 1960 , the number of rediscoveries began to exceed the number of losses; the percentage of the lost comets dropped to two-thirds of its previous value, and at present even to onethird. Eleven comets were discovered again after more than six missed returns (two of them, P/DeVico-Swift and P/Tuttle-Giacobini-Kresák were even rediscovered twice), and 4 comets (P/DeVico-Swift, P/Tempel 1, P/Denning-Fujikawa, and P/Peters-Hartley) after more than ten missed returns.

An earlier analysis of comet losses (Kresák 1981b) has led to the conclusion that there was no persuasive evidence for the actual disappearance of any of the one-apparition comets. Among those of more than one apparition, two-P/Biela and P/Brorsen - were found to have disappeared definitely, P/Neujmin 2 probably, and $\mathrm{P} / \mathrm{Gale}$ possibly. Failure to rediscover P/Neujmin 2 at its very favorable return in 1987 has made its disappearance almost certain.

Among the comets of more than one apparition (Table 4), 7 are classified as lost, and another 6 were missed at the last return. Six of the recently missed returns (P/PerrineMrkos and P/Neujmin 3 in 1982, P/Oterma in 1983, P/Schwassmann-Wachmann 3 in 1985 , P/Wild 1 in 1986, and P/DuToit in 1988) were so unfavorable that the apparent brightness should have remained all the time below magnitude 20 . All the other cases are listed in the upper part of Table 5. $T_{F}$ and $T_{L}$ denote the years of the first and last apparition, respectively. For the suspicious missed returns, which should have been rather bright, the year and the predicted maximum apparent brightness, corrected for the instrumental effects and rounded off to the nearest half-magnitude, are listed. The pairs $\mathrm{T}_{\mathrm{A}}$ (year of perihelion passage) and $\mathrm{M}_{\mathrm{A}}$ (maximum apparent brightness) refer to the missed perihelion passages that were followed by observed ones; the pairs $T_{B}$ and $M_{B}$ to those during and after which the comet has not been observed anymore. The symbol "+" means that the brightness peak should have occurred at a solar elongation less than $30^{\circ}$ and that the given value refers to the position at $\mathrm{E}=30^{\circ}$. This circumstance reduces the discovery probability somewhat. The symbol "-" means that no reliable ephemeris was available and the mean brightness of newly discovered comets was higher at that time, making the observation improbable. The last column of the table demonstrates that it is indeed only $\mathrm{P} / \mathrm{Biela}$ and $\mathrm{P} / \mathrm{Brorsen}$ for which the extinction can be inferred with certainty, and P/Neujmin 2 for which at least a drastic reduction of the absolute brightness must be inferred. The other comets deserve special attention at their future favorable returns. These will occur in this century only for P/Tuttle-Giacobini-Kresák, in 1990, and P/PerrineMrkos, in 1995.

Backward integrations of the orbits of all known short-period comets (Carusi et al. 1985a, Belyaev et al. 1986) have made it possible to identify those prediscovery perihelion passages during which the comets should have been bright and favorably placed. There are 
Table 3. Comets of $P<20$ Years: Discoveries, Losses, and RediscoveriesNumbers/Cumulative Numbers

\begin{tabular}{||ccccc||}
\hline Period & Discoveries & Losses & Rediscoveries & \% Lost \\
\hline $1770-1870$ & $16 / 16$ & $14 / 7$ & 7 & 44 \\
$1871-1900$ & $16 / 32$ & $6 / 11$ & 2 & 34 \\
$1901-1929$ & $16 / 48$ & $16 / 23$ & 4 & 48 \\
$1929-1950$ & $16 / 64$ & $8 / 28$ & 3 & 44 \\
$1951-1970$ & $16 / 80$ & $6 / 24$ & 10 & 30 \\
$1971-1978$ & $16 / 96$ & $1 / 21$ & 4 & 22 \\
$1978-1984$ & $16 / 112$ & $2 / 19$ & 4 & 17 \\
$1984-1988$ & $16 / 128$ & $0 / 19$ & 0 & 15 \\
\hline
\end{tabular}

Table 4. Comets of $P<20$ Years and More Than One Apparition: Discoveries, Losses, and Rediscoveries-Numbers/Cumulative Numbers

\begin{tabular}{|ccccc|}
\hline Period & Discoveries & Losses & Rediscoveries & \% Lost \\
\hline $1770-1854$ & $10 / 10$ & $8 / 3$ & 5 & 30 \\
$1855-1892$ & $10 / 20$ & $7 / 8$ & 2 & 40 \\
$1892-1920$ & $10 / 30$ & $8 / 11$ & 5 & 37 \\
$1921-1937$ & $10 / 40$ & $5 / 14$ & 2 & 35 \\
$1938-1949$ & $10 / 50$ & $5 / 17$ & 2 & 34 \\
$1949-1964$ & $10 / 60$ & $5 / 16$ & 6 & 27 \\
$1965-1974$ & $10 / 70$ & $1 / 11$ & 6 & 16 \\
$1975-1988$ & $11 / 81$ & $2 / 7$ & 6 & 9 \\
\hline
\end{tabular}


indeed several cases for which the absence of observations is surprising and irreconcilable with the present absolute brightness of the comet. This suggests that comets are subject not only to extinction but also to reactivation after a dormant phase (Kresák 1986 and 1987c). The four most striking examples are listed at the bottom of Table 5. Curiously enough, P/Finlay, which experienced the steepest brightness decrease from among all comets observed over 10 or more revolutions (Kresák and Kresáková 1989a), must have been much fainter before the discovery. Even with its mean absolute brightness, it would have been an easy naked-eye object in 1827; with the extrapolated "secular" term, it would have reached first to second magnitude at that time. P/Honda-Mrkos-Pajduśáková and $\mathrm{P} /$ Boethin must have been at least two magnitudes fainter before the discovery than they are today. Otherwise, they could hardly have escaped the attention of comet hunters. P/Denning-Fujikawa, the absolutely faintest periodic comet on record, was probably active only at some returns to the Sun, and at the especially bright apparition in 1881 only after the perihelion passage. Similar intermissions of activity are indicated for $\mathrm{P} / \mathrm{Biela}$ and $\mathrm{P} / \mathrm{Brorsen}$ several revolutions before their final disappearance.

Thus, $\mathrm{P} / \mathrm{Biela}$ and $\mathrm{P} / \mathrm{Brorsen}$ seem to represent the whole net loss of comets by extinction, with the other comets of Table 5 reflecting a balance between transient brightening and fading. These poor statistics do not allow us to say more than that the mean active lifetime of short-period comets is probably between 200 and 500 revolutions. More exactly, this is the inverse extinction probability per revolution, and the actual lifetimes depend on their distribution function. The ultimate extinction tends to be preceded (and thus also predicted) by transient intermissions of activity, extending over several revolutions. These conclusions apply to comets of small perihelion distance, $q<1.5 \mathrm{AU}$. At larger perihelion distances, the apparent brightness of comets is, as a rule, too low to identify the inactive returns reliably, and the number of observed revolutions is too small compared with the potential active lifetimes.

\section{The Final Phases of Aging}

Since comet orbits are unstable, the dynamical end fate of comets should be ejection from the solar system. There is only a very low probability that nongravitational forces could transfer some of them into stable orbits. However, the potential dynamical lifetimes are much longer than the active lifetimes. So the question is what happens to a comet at the end of its last active period? There are three possible answers: (1) a complete disintegration, (2) a complete loss of volatiles, or (3) a complete surface crusting, which does not exclude later reactivation.

Ten years ago there was a common belief that complete disintegration strongly prevailed (Rickman and Vaghi 1976). This was because almost no asteroidal object moving in a typical cometary orbit was known at that time (Kresák 1979). While this still holds for long-period orbits, a number of asteroids moving in orbits indiscernible from those of short-period comets were discovered between 1982 and 1985 . Figure 2 shows the orbits of nine asteroids (above) and nine comets (below) that are most similar to one another. With the possible exception of pair No. 9, 2212 Hephaistos and P/Encke (Galibina and Kastel 1982, Babadzhanov and Obrubov 1983, Napier 1983, Stohl 1986) there is no indication of a genetic association. The pairs were selected simply 
Table 5. Missed Apparitions and Their Computed Maximum Apparent Brightness

\begin{tabular}{|c|c|c|c|c|c|c|}
\hline Comet & $\mathbf{N}_{\mathrm{A}}$ & $\mathrm{T}_{\mathrm{F}}-\mathrm{T}_{\mathrm{L}}$ & $\mathrm{T}_{\mathrm{A}}$ & $\mathrm{M}_{\mathrm{A}}$ & $\mathrm{T}_{\mathrm{B}}$ & $\mathrm{M}_{\mathrm{B}}$ \\
\hline P/Biela & 6 & $1772-1852$ & 1778 & 3.5 & $\begin{array}{l}1886 \\
1886\end{array}$ & $\begin{array}{l}3.5 \\
3.5\end{array}$ \\
\hline P/Brorsen & 5 & $1846-1879$ & 1835 & $4.0+$ & $\begin{array}{l}1890 \\
1940\end{array}$ & $\begin{array}{l}5.0+ \\
4.5+\end{array}$ \\
\hline P/Neujmin 2 & 2 & $1916-1927$ & & & $\begin{array}{l}1971 \\
1987\end{array}$ & $\begin{array}{l}12.5 \\
10.0\end{array}$ \\
\hline P/Gale & 2 & $1927-1938$ & 1893 & $8.5-$ & $\begin{array}{l}1949 \\
1981\end{array}$ & $\begin{array}{l}11.5 \\
14.0+\end{array}$ \\
\hline P/Tempel-Swift & 4 & $1869-1908$ & & & $\begin{array}{l}1950 \\
1982\end{array}$ & $\begin{array}{l}15.5 \\
17.0\end{array}$ \\
\hline P/Tuttle-Giacobini-Kresák & 6 & $1858-1978$ & $\begin{array}{l}1831 \\
1874 \\
1940\end{array}$ & $\begin{array}{r}8.5- \\
10.0- \\
11.0-\end{array}$ & 1984 & 16.5 \\
\hline P/Perrine-Mrkos & 5 & $1896-1968$ & $\begin{array}{l}1869 \\
1922 \\
1942\end{array}$ & $\begin{array}{r}8.5- \\
10.5- \\
10.5-\end{array}$ & 1975 & 16.5 \\
\hline P/DeVico-Swift & 3 & $1844-1965$ & 1833 & $7.0-$ & $\begin{array}{l}1980 \\
1987\end{array}$ & $\begin{array}{l}18.0 \\
18.0\end{array}$ \\
\hline P/Denning-Fujikawa & 2 & $1881-1978$ & $\begin{array}{l}1829 \\
1960 \\
1969\end{array}$ & $\begin{array}{l}5.0 \\
8.5 \\
8.5\end{array}$ & 1987 & 13.5 \\
\hline P/Finlay & 16 & $1886-1988$ & $\begin{array}{l}1827 \\
1873\end{array}$ & $\begin{array}{l}4.5 \\
8.5-\end{array}$ & & \\
\hline P/Honda-Mrkos-Pajdušáková & 7 & $1948-1985$ & $\begin{array}{l}1878 \\
1889 \\
1917 \\
1927\end{array}$ & $\begin{array}{l}7.0+ \\
6.5+ \\
7.0+ \\
7.5+\end{array}$ & & \\
\hline P/Boethin & 2 & $1975-1986$ & $\begin{array}{l}1919 \\
1960\end{array}$ & $\begin{array}{l}7.5 \\
9.0\end{array}$ & & \\
\hline
\end{tabular}



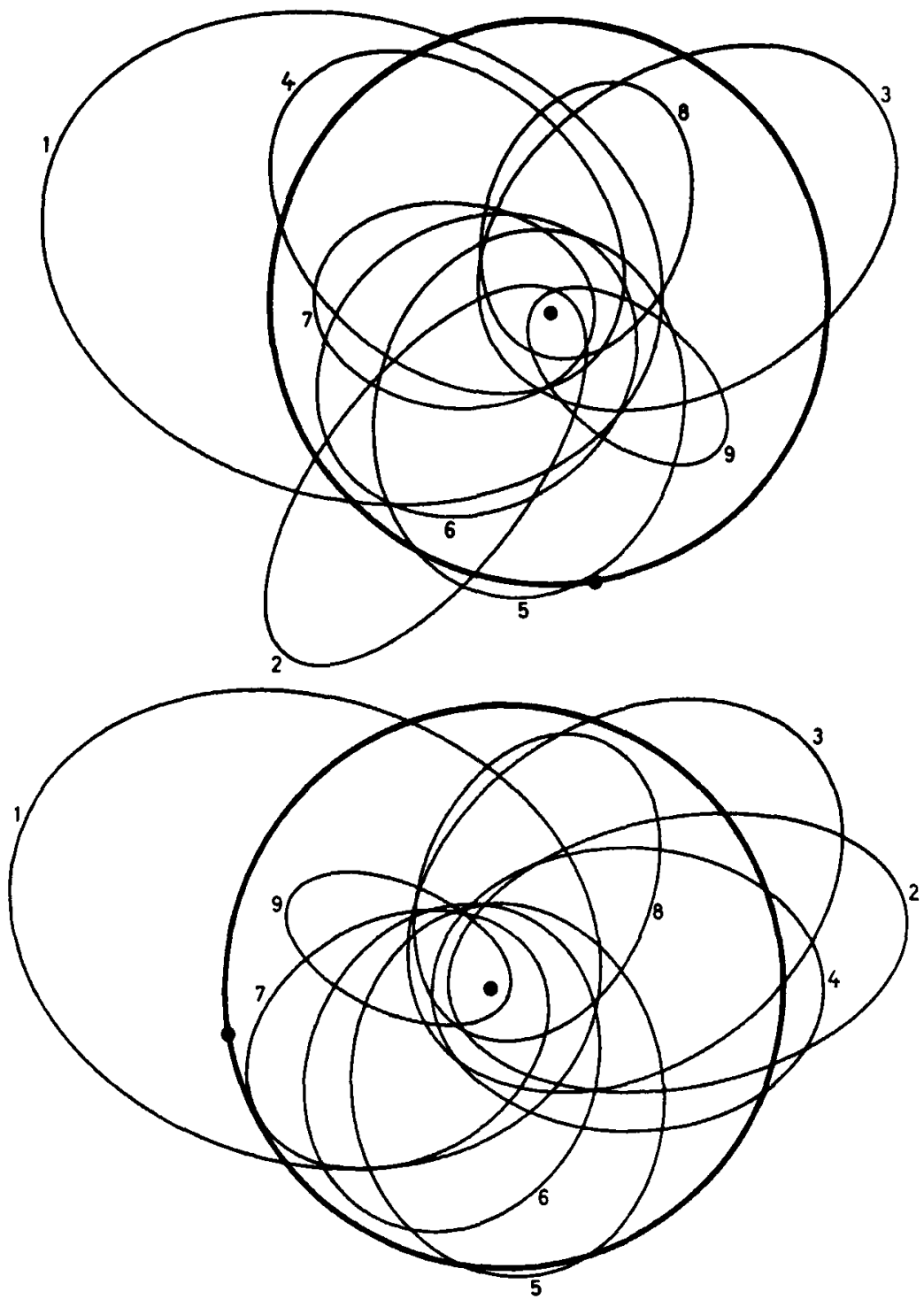

Figure 2. (Top) Most cometlike asteroid orbits: $1=944$ Hidalgo, $2=5025 \mathrm{P}-\mathrm{L}, 3=3552$ $=1983 \mathrm{SA}, 4=1982 \mathrm{YA}, 5=1984 \mathrm{BC}, 6=1983 \mathrm{XF}, 7=1983 \mathrm{LC}, 8=1983 \mathrm{VA}$, and 9 = 2212 Hephaistos. (Bottom) Similar comet orbits: $1=$ P/Wild 1, $2=$ P/DenningFujikawa, 3 = P/Swift-Gehrels, $4=\mathrm{P} /$ Finlay, $5=\mathrm{P} / \mathrm{Kopff}, 6=\mathrm{P} /$ Tempel 1, $7=$ $\mathrm{P} /$ Blanpain, $8=\mathrm{P} /$ Grigg-Skjellerup, and $9=\mathrm{P} /$ Encke. The two pictures are rotated by $90^{\circ}$ with respect to one another, as indicated by the dots marking the Sun and the perihelion of Jupiter's orbit (the thick, low-eccentricity ellipse). The vernal equinox is down for the asteroids and to the left for the comets (Kresák 1985). 
according to their proximity in the a/e diagram (Kresák 1979 and 1985), which, for similar or low inclinations, also implies a similar value of the Tisserand invariant.

Recent perturbation computations by Milani et al. (1989), extending over 200,000 years, classify seven of the known Apollo/Amor objects as "comets." All of them are included in Figure 2, together with two additional objects: 944 Hidalgo and 1983 LC. Hidalgo was not considered by Milani et al. because of its large perihelion distance, $q=2.0$ AU. However, according to Kozai (1979), this should decrease to $1.1 \mathrm{AU}$ at the extreme of the libration cycle of the argument of perihelion by a mechanism affecting the motion of some comets (including P/Halley). The orbit of $1983 \mathrm{LC}$ is very poorly determined, being based on only 12 observations over a period of 19 days. It must be stressed as well that the observing arcs of the other objects, less than 100 days for five of them, put constraints on the reliability of the long-term perturbation computations-in particular, on the occurrence of resonances and close encounters with Jupiter. Thus, the results have mainly a statistical character, and the original hyperbolic orbits of $1983 \mathrm{XF}$ and 1984 BC mean only that they may have been captured from long-period comet-like orbits not long ago.

Assuming these objects have albedos similar to that of $\mathrm{P} / \mathrm{Halley}$, the diameters of most of the objects range between 2 and $10 \mathrm{~km}$. Exceptions are 944 Hidalgo, with a diameter of about $30 \mathrm{~km}$, and $1983 \mathrm{LC}$ and $5025 \mathrm{P}-\mathrm{L}$, with diameters smaller than $1 \mathrm{~km}$. This size distribution indicates that our current list of such objects is far from complete in the range of typical diameters of short-period comet nuclei.

An earlier classification based on the elliptic restricted three-body (Sun-Jupiterasteroid) problem (Hahn and Rickman 1985, Rickman 1985) lists 944 Hidalgo, 1983 SA, $1983 \mathrm{XF}$, and $1984 \mathrm{BC}$ as first-rank candidates for objects of cometary origin, and 1982 YA, 1983 LC, and 1983 VA as only somewhat less certain cases.

Using other than dynamical arguments, a cometary origin has also been suggested for some other asteroids. In particular, these were 2201 Oljato, due to its unique spectral properties (McFadden et al. 1984) and interplanetary magnetic field disturbances recorded in its vicinity by the Pioneer-Venus spacecraft (Russell et al. 1984), and 3200 Phaeton, which may be the parent object of the Geminid meteor stream (Whipple 1983b). While the Geminid meteors do not show any definite differences from those produced by periodic comets (Jacchia et al. 1965), it appears impossible to explain the small aphelion distance of Phaeton, 2.4 AU, by a cometary origin. Associations of much less intense, and sometimes questionable, meteor showers with other Apollo objects have been suggested by Sekanina (1976), Drummond (1982), Babadzhanov and Obrubov (1983), and Olsson-Steel (1987a, 1987b, 1988). The discovery of the asteroid dust bands by the Infrared Astronomical Satellite (IRAS) (Dermott et al. 1985) has provided evidence that the asteroids can also produce large amounts of interplanetary dust.

Thus, the dynamical properties of the inactive objects represent a more reliable criterion for their origin than any other indirect evidence. It appears that there are a considerable number of inactive objects moving in cometary orbits. Whether the absence of activity represents these objects' final evolutionary stage after complete depletion of volatiles, or whether it is only temporary, is still an open problem.

The primary criterion for classifying and cataloging an object as a comet or an asteroid is its appearance. Based on this, 17 periodic comets have already received provisional asteroid designations: P/Smirnova-Chernych even four times, and P/Whipple, P/Reinmuth 1, and P/Urata-Niijima twice. These identifications, mainly due to the 
computations by Nakano $(1984,1987,1988$, and 1989), demonstrate that the discrimination is not always easy. All these objects have shown definite cometary activity on other occasions, and records of their asteroidal appearance come from moderate heliocentric distances: mostly between 2.0 and $2.7 \mathrm{AU}$, and for P/Väisälä 2 and P/UrataNiijima, even within 1.5 AU. For the problem of marginal activity and reflectance spectra, see Degewij and Tedesco (1982).

There is one more cataloged asteroid that was evidently a comet: $1939 \mathrm{TN}$, discovered by Oterma (see Marsden and Bardwell 1982). It is also noteworthy that as many as eight of the nine asteroids plotted in Figure 2 were discovered by observers who have also discovered a number of comets, using the same instruments (Baade, Gehrels, Chernykh, Wild, Helin, Bowell, and IRAS). This demonstrates that the claimed asteroidal appearance did not result from overlooked diffuseness, but was a real characteristic of these objects.

An entirely unique object is 2060 Chiron, with a perihelion distance as large as 13.45 AU. For comparison, the extreme perihelion distances of all the other observed interplanetary objects were $5.15 \mathrm{AU}$ for the asteroids, $5.54 \mathrm{AU}$ for the short-period comets, and 6.88 AU for the long-period comets. Recent observations (Meech and Belton 1989 , and Meech, this book) have demonstrated beyond a doubt the cometary nature of Chiron. Long-term integrations (Oikawa and Everhart 1979) have shown that its chaotic orbital evolution resembles the way in which long-period comets may be stepwise captured into short-period orbits, and that Chiron can reach this stage within the next $10^{5}$ years. If so, its extraordinary size (estimated at 200 to $300 \mathrm{~km}$ in diameter) would predestine it to be an extremely bright object, incomparable with anything seen before, for our remote descendants.

The present situation does not exclude any of the three possible end fates of periodic comets. There are cases of total, or almost total, disintegration-P/Biela, for example. There are also totally inactive objects, for which it is impossible to tell whether they still have some supply of volatiles for a future reactivation. Therefore, asteroids moving in comet-like orbits deserve special attention of observers-both in order to improve the accuracy of the objects' orbits and to assess whether they are indeed totally inactive. The same holds for attempts to rediscover the lost periodic comets.

\section{Bibliography and References}

Andrienko, D.A., and Karpenko, A.V. (1987). Fizicheskie kharakteristiki komet 19761980 gg., Nauka, Moskva.

Babadzhanov, P.B., and Obrubov, Yu.V. (1983). "Secular perturbations of Apollo, Amor and Aten asteroid orbits and theoretical radiants of meteor showers probably associated with them," in C.-I. Lagerkvist and H. Rickman (eds.), Asteroids, Comets, Meteors, Univ. Uppsala, 411-417.

Bailey, M.E. (1983). "The structure and evolution of the solar system comet cloud," Mon. Not. Roy. Astron. Soc. 204, 603-633.

Bailey, M.E., Clube, S.V.M., and Napier, W.M. (1986). "The origin of comets," Vistas Astron. 29, 52-112.

Belyaev, N.A., Kresák, Ľ., Pittich, E.M., and Pushkarev, A.N. (1986). Catalogue of Short-Period Comets, Veda, Bratislava. 
Bobrovnikoff, N.T. (1941). "Investigations of the brightness of comets, Part I," Contrib. Perkins Obs. 15, 49-187.

Bobrovnikoff, N.T. (1942). "Investigations of the brightness of comets, Part II," Contrib. Perkins Obs. 16, 189-300.

Carusi, A., Kresák, Ľ., Perozzi, E., and Valsecchi, G.B. (1985a). Long-Term Evolution of Short-Period Comets, A. Hilger, Bristol.

Carusi, A., Kresák, L., Perozzi, E., and Valsecchi, G.B. (1985b). "First results of the integration of motion of short-period comets over 800 years," in A. Carusi and G.B. Valsecchi (eds.), Dynamics of Comets: Their Origin and Evolution, IAU Coll. 83, D. Reidel, Dordrecht, 319-340.

Carusi, A., Kresák, Ľ., Perozzi, E., and Valsecchi, G.B. (1987). "Long-term resonances and orbital evolutions of Halley-type comets," in Z. Ceplecha and P. Pecina (eds.), Interplanetary Matter, ERAM 10, Publ. Astron. Inst. Czechosl. Acad. Sci. 67, 29-32.

Clube, S.V.M., and Napier, V.M. (1984). "Comet capture from molecular clouds: A dynamical constraint on star and planet formation," Mon. Not. Roy. Astron. Soc. 208, 575-588.

Crifo, J.F. (1987). "Are cometary dust mass loss rates deduced from optical emissions reliable?", in Z. Ceplecha and P. Pecina (eds.), Interplanetary Matter, ERAM 10, Publ. Astron. Inst. Czechosl. Acad. Sci. 67, 59-66.

Degewij, J., and Tedesco, E.F. (1982). "Do comets evolve into asteroids? Evidence from physical studies," in L.L. Wilkening and M.S. Matthews (eds.), Comets, IAU Coll. 61, Univ. Arizona, Tucson, 665-695.

Delsemme, A.H. (1976). "Can comets be the only source of interplanetary dust?", in H. Elsässer and H. Fechtig (eds.), Interplanetary Dust and Zodiacal Light, IAU Coll. 31, Lecture Notes in Physics 48, 481-484.

Dermott, S.F., Nicholson, P.D., Burns, J.A., and Houck, J.R. (1985). "An analysis of IRAS solar system dust bands," in R. Giese and P. Lamy (eds.), Properties and Interactions of Interplanetary Dust, IAU Coll. 85, D. Reidel, Dordrecht, 395-409.

Dobrovolsky, O.V., Ibadinov, Kh.I., Aliev, S., and Gerasimenko, S.I. (1986). "Thermal regime and surface structure of periodic comet nuclei," in B. Battrick, E.J. Rolfe, and R. Reinhard (eds.), Exploration of Halley's Comet, ESA SP-250, Vol. 2, 389-394.

Drummond, J.D. (1982). "Theoretical meteor radiants of Apollo, Amor, and Aten asteroids," Icarus 49, 143-153.

Duncan, M., Quinn, T., and Tremaine, S. (1987). "The formation and extent of the solar system comet cloud," Astron. J. 94, 1330-1338.

Everhart, E. (1976). "The evolution of comet orbits," in B. Donn, M. Mumma, W. Jackson, M. A'Hearn, and R. Harrington (eds.), The Study of Comets, IAU Coll. 25, NASA SP-393, 445-464.

Everhart, E. (1977). "The evolution of comet orbits as perturbed by Uranus and Neptune," in A.H. Delsemme (ed.), Comets, Asteroids, Meteorites, IAU Coll. 39, Univ. Toledo, 99-104.

Fernández, J.A. (1981). "New and evolved comets in the solar system," Astron. Astrophys. 96, 26-35.

Fernández, J.A. (1985a). "The formation and dynamical survival of the comet cloud," in A. Carusi and G.B. Valsecchi (eds.), Dynamics of Comets: Their Origin and Evolution, IAU Coll. 83, D. Reidel, Dordrecht, 45-70. 
Fernández, J.A. (1985b). "Dynamical capture and physical decay of short-period comets," Icarus 64, 308-319.

Fernández, J.A., and Ip, W.-H. (1983). "On the time evolution of the cometary influx into the region of the terrestrial planets," Icarus 54, 377-387.

Galibina, I.V., and Kastel, G.R. (1982). "On possible association of the asteroid 2212 - 1978 SB with the comet Encke," Komety i meteory 33, 45-46.

Green, S.F., McDonnell, J.A.M., Perry, C.H., Nappo, S., and Zarnecki, J.C. (1987). "P/Halley dust coma: Grains or rocks?", in E.J. Rolfe and B. Battrick (eds.), Diversity and Similarity of Comets, ESA SP-278, 379-384.

Guliev, A.S., and Bajramov, A.Sh. (1988). "New statistical approach to the problem of cometary brightness fading," Kinematika i fizika nebesnykh tel 4, 30-34.

Hahn, G., and Rickman, H. (1985). "Asteroids in cometary orbits," Icarus 61, 417-442.

Hajduk, A. (1986). "Debris from comet Halley and the risk for space probes," in C.-I. Lagerkvist, B.A. Lindblad, H. Lundstedt, and H. Rickman (eds.), Asteroids, Comets, Meteors II, Univ. Uppsala, 497-500.

Hills, J.G. (1981). "Comet showers and the steady state infall of comets from the Oort cloud," Astron. J. 86, 1730-1740.

Holetschek, J. (1916). "Untersuchungen über die Grösse und Helligkeit der Kometen und ihrer Schweife, IV," Wiener Denkschrifte math. nat. Klasse 93, 1-105.

Holetschek, J. (1917). "Untersuchungen über die Grösse und Helligkeit der Kometen und ihrer Schweife, V," Wiener Denkschrifte math. nat. Klasse 94, 1-114.

Hughes, D.W. (1975). "Cometary outbursts: A brief survey," Quart. Roy. Astron. Soc. $16,410-427$.

Hughes, D.W., and Daniels, P.A. (1983). "The secular variation of cometary magnitude," Icarus 53, 444-452.

Jacchia, L.G., Verniani, F., and Briggs, R.E. (1967). "An analysis of the atmospheric trajectories of 413 precisely reduced photographic meteors," Smithson. Contr. Astrophys. 10, 1-139.

Joss, P.C. (1973). "On the origin of short-period comets," Astron. Astrophys. 25, 271273.

Keller, H.U. (1987). "The nucleus of comet Halley," in E.J. Rolfe and B. Battrick (eds.), Diversity and Similarity of Comets, ESA SP-278, 447-454.

Kozai, Y. (1979). "Secular perturbations of asteroids and comets," in R.L. Duncombe (ed.), Dynamics of the Solar System, IAU Symp. 81, 231-237.

Kresák, L. (1966). "On two aspects of evolution of short-period comets," in P. Swings (ed.), Nature et Origine des Comètes, Mém. Soc. Roy. Sci. Liège, Ser. 5, 12, 459467.

Kresák, Ľ. (1974). "The aging and the brightness decrease of comets," Bull. Astron. Inst. Czechosl. 25, 87-112.

Kresák, Ľ. (1979). "Dynamical interrelations among comets and asteroids," in T. Gehrels and M.S. Matthews (eds.), Asteroids, Univ. Arizona, Tucson, 289-309.

Kresák, L. (1980). "Sources of interplanetary dust," in I. Halliday and B.A. McIntosh (eds.), Solid Particles in the Solar System, IAU Symp. 90, 211-222.

Kresák, L. (1981a). "Evolutionary aspects of the splits of cometary nuclei," Bull. Astron. Inst. Czechosl. 32, 19-40.

Kresák, Ľ. (1981b). "The lifetimes and disappearance of periodic comets," Bull. Astron. Inst. Czechosl. 32, 321-339. 
Kresák, Ľ. (1982). "Comet discoveries, statistics, and observational selection,” in L.L. Wilkening and M.S. Matthews (eds.), Comets, IAU Coll. 61, Univ. Arizona, Tucson, 56-82.

Kresák, L. (1984). "The lifetimes and disappearance of long-period comets," Bull. Astron. Inst. Czechosl. 35, 129-150.

Kresák, L. (1985). "The aging and lifetimes of comets," in A. Carusi and G.B. Valsecchi (eds.), Dynamics of Comets: Their Origin and Evolution, IAU Coll. 83, D. Reidel, Dordrecht, 279-302.

Kresák, L. (1986). "On the aging process of periodic comets," in B. Battrick, E.J. Rolfe, and R. Reinhard (eds.), Exploration of Halley's Comet, ESA SP-250, Vol. 2, 433 438.

Kresák, Ľ. (1987a). "The systems of interplanetary objects," in M. Fulchignoni and Ľ. Kresák (eds.), The Evolution of the Small Bodies of the Solar System, Proc. Internat. School Phys. Enrico Fermi 98, North-Holland, Amsterdam, 10-32.

Kresák, L. (1987b). "The 1808 apparition and the long-term physical evolution of periodic comet Grigg-Skjellerup," Bull. Astron. Inst. Czechosl. 38, 65-75.

Kresák, Ľ. (1987c). "Dormant phases in the aging of periodic comets," Astron. Astrophys. 187, 906-908.

Kresák, L., and Kresáková, M. (1987a). "The absolute total magnitude of periodic comets and their variations," in E.J. Rolfe and B. Battrick (eds.), Diversity and Similarity of Comets, ESA SP-278, 37-42.

Kresák, Ľ ., and Kresáková, M. (1987b). "The contribution of periodic comets to the Zodiacal cloud," in Z. Ceplecha and P. Pecina (eds.), Interplanetary Matter, ERAM 10, Publ. Astron. Inst. Czechosl. Acad. Sci. 67, 265-271.

Kresák, Ĺ., and Kresáková, M. (1989a). "The absolute magnitudes of periodic comets, I and II," Bull. Astron. Inst. Czechosl. 40, 269-284, and in press.

Kresák, Ľ., and Kresáková, M. (1989b). "On the secular brightness decrease of periodic comets," Icarus, in press.

Kuiper, G.P. (1951). "On the origin of the solar system," in J.A. Hynek (ed.), Astrophysics, McGraw-Hill, New York, 357-427.

Marsden, B.G. (1985). "Nongravitational forces in comets: The first fifteen years," in A. Carusi and G.B. Valsecchi (eds.), Dynamics of Comets: Their Origin and Evolution, IAU Coll. 83, D. Reidel, Dordrecht, 343-352.

Marsden, B.G., and Bardwell, C.M. (1982). Catalogue of Orbits of Unnumbered Minor Planets, IAU Minor Planet Center, Cambridge.

Marsden, B.G., and Roemer, E. (1982). "Basic information and references," in L.L. Wilkening and M.S. Matthews (eds.), Comets, IAU Coll. 61, Univ. Arizona, Tucson, 707-733.

McDonnell, J.A.M., Kissel, J., Grün, E., Grard, R.J.L., Langevin, Y., Olearczyk, R.E., Perry, C.H., and Zarnecki, J.C. (1986). "Giotto's dust impact detection system DIDSY and particulate impact analyser PIA: Interim assessment of the dust distribution and properties within the coma," in B. Battrick, E.J. Rolfe, and R. Reinhard (eds.), Exploration of Halley's Comet, ESA SP-250, Vol. 2, 25-38.

McFadden, L.A., Gaffey, M.J., and McCord, T.B. (1984). "Mineralogical-petrological characterization of near-Earth asteroids," Icarus 59, 25-40.

Meech, K.J., and Belton, M.J.S. (1989). “2060 Chiron,” IAU Circ. No. 4770. 
Meisel, D.D., and Morris, C.S. (1976). "Comet brightness parameters: Definition, determination and correlation," in B. Donn, M. Mumma, W. Jackson, M. A'Hearn, and R. Harrington, The Study of Comets, IAU Coll. 25, NASA SP-393, 410-444.

Meisel, D.D., and Morris, C.S. (1982). "Comet head photometry: Past, present, and future," in L.L. Wilkening and M.S. Matthews (eds.), Comets, IAU Coll. 61, Univ. Arizona, Tucson, 413-432.

Milani, A., Carpino, M., Hahn, G., and Nobili, A.M. (1989). "Dynamics of planetcrossing asteroids: Classes of orbital behavior. Project Spaceguard," Icarus 78, 212269.

Morris, C.S. (1973). “On aperture corrections for comet magnitude estimates," Publ. Astron. Soc. Pacific 85, 470-473.

Nakano, S. (1984). "Identifications of comets and minor planets," Japan Astron. Circ. No. 409.

Nakano, S. (1987). "Identifications with comets," Minor Planet Circ. 12025.

Nakano, S. (1988). "Identifications with a comet," Minor Planet Circ. 12626.

Nakano, S. (1989). "Identifications with comets," Minor Planet Circ. 14384.

Napier, W.M. (1983). "The orbital evolution of short period comets," in C.-I. Lagerkvist and H. Rickman (eds.), Asteroids, Comets, Meteors, Univ. Uppsala, 391-396.

Oikawa, S., and Everhart, E. (1979). "Past and future orbit of 1977 UB, object Chiron," Astron. J. 84, 134-139.

Olsson-Steel, D. (1987a). "Asteroid 5025 P-L, comet 1967 II Rudnicki, and the Taurid meteoroid complex," Observatory 107, 157-160.

Olsson-Steel, D. (1987b). "Meteoroid streams associated with Apollo asteroids: Evidence from the Adelaide radar orbit surveys," in Z. Ceplecha and P. Pecina (eds.), Interplanetary Matter, ERAM 10, Publ. Astron. Inst. Czechosl. Acad. Sci. 67, 125129.

Olsson-Steel, D. (1988). "Identification of meteoroid streams from Apollo asteroids in the Adelaide radar orbit surveys," Icarus 75, 64-96.

Pons, J.L. (1829). "Notizen über den 1808 am 6ten Februar von Pons entdeckten Cometen," Astron. Nachr. 7, 113.

Rickman, H. (1985). "Interrelations between comets and asteroids," in A. Carusi and G.B. Valsecchi (eds.), Dynamics of Comets: Their Origin and Evolution, IAU Coll. 83, D. Reidel, Dordrecht, 149-172.

Rickman, H. (1986). "Masses and densities of comets Halley and Kopff," in O. Melita (ed.), Comet Nucleus Sample Return, ESA SP-249, 195-205.

Rickman, H., and Vaghi, S. (1976). "A Monte Carlo simulation of the orbital evolution of comets in the inner planetary region," Astron. Astrophys. 51, 327-342.

Rickman, H., Kamél, L., Festou, M.C., and Froeschlé, C. (1987). "Estimates of masses, volumes and densities of short-period comet nuclei," in E.J. Rolfe and B. Battrick (eds.), Diversity and Similarity of Comets, ESA SP-278, 471-481.

Russell, C.T., Aroian, R., Arghavani, M., and Nock, K. (1984). "Interplanetary magnetic field enhancements and their association with the asteroid 2201 Oljato," Science 226, 43-45.

Sekanina, Z. (1964). "Secular variations in the absolute brightness of short-period comets," Bull. Astron. Inst. Czechosl. 15, 1-16.

Sekanina, Z. (1976). "Statistical model of meteor streams, IV. A study of radio streams from the synoptic year," Icarus 27, 265-321. 
Sekanina, Z. (1982). "The problem of split comets in review," in L.L. Wilkening and M.S. Matthews (eds.), Comets, IAU Coll. 61, Univ. Arizona, Tucson, 251-287.

Sekanina, Z. (1984). "Disappearance and disintegration of comets," Icarus 58, 81-100.

Shulman, L.M. (1972). "The evolution of cometary nuclei," in G.A. Chebotarev, E.I. Kazimirchak-Polonskaya, and B.G. Marsden (eds.), The Motion, Evolution of Orbits, and Origin of Comets, IAU Symp. 45, 271-276.

Štohl, L. (1986). “On meteor contribution by short-period comets," in B. Battrick, E.J. Rolfe, and R. Reinhard (eds.), Exploration of Halley's Comet, ESA SP-250, Vol. 2, 225-228.

Štohl, L. (1987). "On meteor contribution by short-period comets," Astron. Astrophys. 187, 933-934.

Svoren, J. (1979). "Secular variations in the absolute brightness of periodic comets," Contr. Astron. Obs. Skalnaté Pleso 8, 105-140.

Vsekhsvyatskij, S.K. (1958). Fizicheskie kharakteristiki komet, Fizmatgizdat, Moskva. English translation: Physical Characteristics of Comets, Israel Program for Scientific Translations, Jerusalem, 1964.

Vsekhsvyatskij, S.K. (1966). Fizicheskie kharakteristiki komet nablyudavshikhsya v 1954-1960 gg., Nauka, Moskva. Abbreviated English translation: Sov. Astron. 6, 849-854.

Vsekhsvyatskij, S.K. (1967). Komety 1961-1965 gg., Nauka, Moskva. Abbreviated English translation: Sov. Astron. 10, 1034-1041.

Vsekhsvyatskij, S.K. (1972). "Cometary observations and variations in cometary brightness," in G.A. Chebotarev, E.I. Kazimirchak-Polonskaya, and B.G. Marsden (eds.), The Motion, Evolution of Orbits, and Origin of Comets, IAU Symp. 45, 9-15.

Vsekhsvyatskij, S.K. (1979). Fizicheskie kharakteristiki komet 1971-1975 gg., Naukova Dumka, Kiev.

Vsekhsvyatskij, S.K., and Ilchischina, N.I. (1974). Fizicheskie kharakteristiki komet 1965-1970 gg., Nauka, Moskva. Abbreviated English translation: Sov, Astron. 15, 310-313.

Weissman, P.R. (1982). "Dynamical history of the Oort cloud," in L.L. Wilkening and M.S. Matthews (eds.), Comets, IAU Coll. 61, Univ. Arizona, Tucson, 637-658.

Weissman, P.R. (1986). "The Oort cloud in transition," in C.-I. Lagerkvist, B.A. Lindblad, H. Lundstedt, and H. Rickman (eds.), Asteroids, Comets, Meteors II, Univ. Uppsala, 197-206.

Whipple, F.L. (1964). "Evidence for a comet belt beyond Neptune," Proc. Natl. Acad. Sci. 51, 711-718.

Whipple, F.L. (1967). "On maintaining the meteoritic complex," in J.L. Weinberg (ed.), The Zodiacal Light and the Interplanetary Medium, NASA SP-150, 409-426.

Whipple, F.L. (1972). "The origin of comets," in G.A. Chebotarev, E.I. KazimirchakPolonskaya, and B.G. Marsden (eds.), The Motion, Evolution of Orbits, and Origin of Comets, IAU Symp. 45, 401-409.

Whipple, F.L. (1978a). "Comets," in J.A.M. McDonnell (ed.), Cosmic Dust, J. Wiley, Chichester, 1-73.

Whipple, F.L. (1978b). "Cometary brightness variation and nucleus structure," Moon and Planets 18, 343-359.

Whipple, F.L. (1980). "Rotation and outbursts of comet P/Schwassmann-Wachmann 1," Astron. J. 85, 305-313. 
Whipple, F.L. (1983a). "Comets: Nature, evolution, and decay," in R.M. West (ed.), Highlights of Astronomy 6, D. Reidel, Dordrecht, 323-331.

Whipple, F.L. (1983b). "1983 TB and the Geminid meteors," IAU Circ. No. 3881.

Whipple, F.L. (1984). "Comet P/Holmes 1892 III - A case of duplicity?", Icarus 60, 522-531.

Whipple, F.L. (1986). "The cometary nucleus: Current concepts," in B. Battrick, E.J. Rolfe, and R. Reinhard (eds.), Exploration of Halley's Comet, ESA SP-250, Vol. 2, 281-288.

Whipple, F.L. (1987). "The cometary nucleus: Current concepts," Astron. Astrophys. $187,852-858$.

Whipple, F.L., and Douglas-Hamilton, D.H. (1966). "Brightness changes in periodic comets," in P. Swings (ed.), Nature et Origine des Comètes, Mém. Soc. Roy. Sci. Liège, Ser. $5,12,469-480$.

Whipple, F.L., and Hamid, S.E. (1972). "A search for Encke's comet in ancient Chinese records: A progress report," in G.A. Chebotarev, E.I. Kazimirchak-Polonskaya, and B.G. Marsden (eds.), The Motion, Evolution of Orbits, and Origin of Comets, IAU Symp. 45, 152-154. 